\title{
Low-temperature vibration characteristics in InSe single crystals intercalated by $\mathrm{Ni}$
}

\author{
A. Baran ${ }^{1}$, M. Botko ${ }^{2}$, M. Kajňaková ${ }^{2}$, A. Feher ${ }^{2}$, S. Feodosyev ${ }^{3}$, E. Syrkin ${ }^{3}$, M. Klochko ${ }^{3}$, \\ N. Tovstyuk ${ }^{4,5}$, I. Grygorchak ${ }^{5}$, and V. Fomenko ${ }^{4}$ \\ ${ }^{1}$ Department of Physics, Faculty of Electrical Engineering and Informatics, Technical University of Košice \\ Park Komenského 2, Košice 04200, Slovakia \\ 2 Institute of Physics Faculty of Science, P.J. Šafárik University in Košice \\ Park Angelinum 9, Košice 04154, Slovakia \\ ${ }^{3}$ B. Verkin Institute for Low Temperature Physics and Engineering of the National Academy of Sciences of Ukraine \\ 47 Lenin Ave., Kharkov 61103, Ukraine \\ E-mail: feodosiev@ilt.kharkov.ua \\ ${ }^{4}$ Ivan Franko Lviv National University, 50 Dragomanova Str., Lviv 79005, Ukraine \\ ${ }^{5}$ National University “Lvivska Politechnika”, 12 St. Bandery Str., Lviv 79000, Ukraine
}

Received May 12, 2015, published online September 25, 2015

\begin{abstract}
The theoretical and experimental study of low-temperature heat capacity of InSe intercalated by nickel as a function of temperature has been carried out in range $0.3-30 \mathrm{~K}$. The effect of intercalation by nickel on phonon heat capacity $\left(C_{\mathrm{ph}}\right)$ of InSe has been investigated. Two peaks observed in relative change of phonon specific heat
\end{abstract} $\left(\Delta C_{\mathrm{ph}} / C_{\mathrm{ph}}\right)$ suggested non-Debye excess in heat capacity for all intercalated $\mathrm{Ni}_{X}$ InSe systems.

PACS: 63.20.-e Phonons in crystal lattices;

63.22.Np Layered systems;

63.20.kp Phonon-defect interactions.

Keywords: layered single crystals, intercalated compounds, heat capacity Debye and Einstein models.

\section{Introduction}

Among wide range of strong anisotropy single crystals, layered single crystals are very important, both from the point of view of fundamental studies [1,2] and technical application [3]. Semiconductor layered crystals belong to them [4]. In the last century, active studying of these crystals was caused by both hypothesis of the realization of high-temperature superconductivity [5] and their practical usage as renewable energy storages [3,6] or in systems of hydrogen accumulation [7]. Nowadays these crystals are the objects of great interest for the creation of nanoannealed N-barrier structures [8].

Intercalation of these semiconductors allows them to create a wide range of new compounds with unique properties and features. Intercalation with magnetoactive components leads to the construction of structures with alternating semiconducting and magnetic active layers, which can be interesting for spintronics, because they provide a prin- cipal possibility of optical or electrical guidance of the magnetic properties [6].

InSe compound belongs to the group of layered semiconductors with strong anisotropy. It is so due to the strong covalent bonds within the layers and the weak van der Waals forces between the layers. Therefore, InSe has also attracted significant interest as a host for intercalation because of its capacity, like other layered compounds, to insert different foreign atoms into its van der Waals gap. The unusual optical, transport and magnetic properties have been discovered in layered materials [1,2], which is the result of presence of the strong anisotropy of chemical bond, 2D conductivity and indirect exchange interaction between magnetic ions via two-dimensional electronic gas.

In many disordered solid materials, such as glasses, polymers, biological media, or nanoparticles an unusual and unique feature, a so-called "boson peak" (BP), was observed in neutron [9,10] and x-ray [11,12] scattering experiments, as well as in absorption spectra in the $\mathrm{THz}$ 
region [13,14] and in calorimetric measurements [15-17]. $\mathrm{BP}$ is an anomalous enhanced vibrational density of states in the low-frequency spectral range over that predicted by Debye. It is generally accepted that BP is associated with some sort of disorder (lattice disorder on a microscopic level, random crystalline systems), but it apparently manifests differently depending on the nature of the system. Despite the extensive study of BP, physical origin of BP has remained unclear. It is still a matter of discussion. A lot of theories have been constructed to explain the microscopic origin of the BP [18-27], which attributed it to different types of vibrational excitations. Some of them ascribe the BP to a strongly localized excitations induced by the intrinsic disorder, whereas others attribute it to the collective propagating modes.

In this paper, the low-temperature heat capacity of $\beta$-InSe single crystals, pure and intercalated by $\mathrm{Ni}$ with concentration $x=0.25,0.5,0.75,1,3,5,7$ and 10 at.\% were studied both from theoretical and experimental points of view. The anomalous behavior in temperature dependence of the relative change of heat capacity $\left(\Delta C_{\mathrm{ph}} / C_{\mathrm{ph}}\right)$ was observed for all intercalated $\mathrm{Ni}_{x} \mathrm{InSe}$ compounds. The results of the theoretical analysis correspond to the experimental measurements.

\section{Experimental methods and characterization of samples}

The synthesis of intercalated $\mathrm{Ni}_{x} \operatorname{InSe}(x=0,0.25,0.5$, $0.75,1,3,5,7$ and 10 at.\%) compounds has been carried out as a direct synthesis of the components in the quartz ampoules, pumped out to the $10 \mathrm{~Pa}$ pressure of the residual gas. One vibrational mixing has been used in order to make the alloy homogeneous.

Compounds of superhigh cleanness were taken. In order to avoid the creation of nickel selenides, the synthesis has been carried out during 72 hours at temperature not higher than $960 \mathrm{~K}$. Then, single crystals have been grown from the synthesized compounds using the Bridgmen-Stockbarger method. The velocity of ampoule dropping was $3 \mathrm{~mm} / \mathrm{cm}$ at temperature gradient in the vicinity of crystal growing $30 \mathrm{deg} / \mathrm{cm}$. In order to avoid irregular impurity distribution in single crystal and to accelerate the process of the impurity transition into the solid solution [28] the grown crystals have been thermal treated in evacuated quartz ampoules at $870 \mathrm{~K}$ during 60 hours.

In this work, the single crystal $\beta$-InSe was intercalated with different $\mathrm{Ni}$ concentration. Let us remind that $\beta$-InSe looks like a set of sandwiches of monoatomic layers Se-In-In-Se bonded by weak van der Waals intercalation along $c$ axis (Fig. 1). All our investigations were performed with single crystal samples of $\beta$-InSe of hexagonal space group $P 6_{3} / m m c$ (194) with two sandwiches of Se-In-In-Se ( 8 atoms) in one unit cell with lattice parameters $a=b=0.40026 \mathrm{~nm}, c=1.66426 \mathrm{~nm}$ [29].

The x-ray studies have been performed on DRON-4-07 diffractometer $\left(\mathrm{Cu} K_{\alpha}\right.$ radiation, step $0,05^{\circ}$, angular range

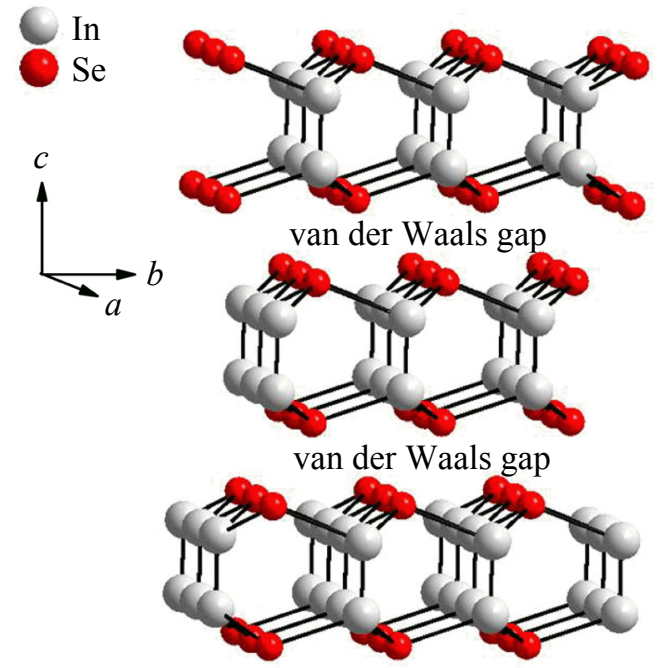

Fig. 1. (Color online) Crystal structure of $\beta$-InSe single crystal.

$8-135^{\circ}$ ). The structural characteristics of $\mathrm{Ni}_{x}$ InSe were calculated using the Crysfire program, with parameters refined by the least squares method. The diffraction patterns of the single crystal InSe both pure and intercalated by nickel with different concentrations are given in Fig. 2 . The diffraction patterns are indexed in the hexagonal space group $P 6_{3} / m m c(194)[29,30]$. The $\mathrm{x}$-ray phase analysis has shown that the material obtained is homogeneous. Neither substitutional phases of InSe compounds nor nickel were found in the free state.
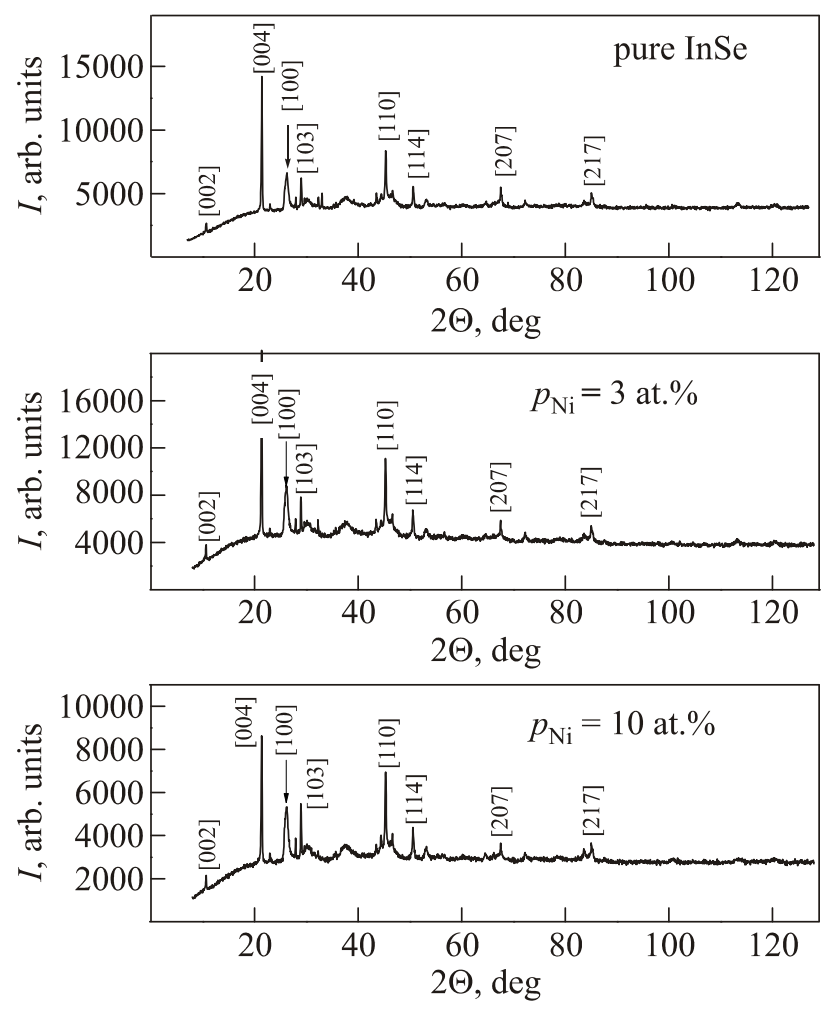

Fig. 2. Diffraction patterns of the single crystal InSe both pure and intercalated by nickel with different $\mathrm{Ni}$ concentrations. 


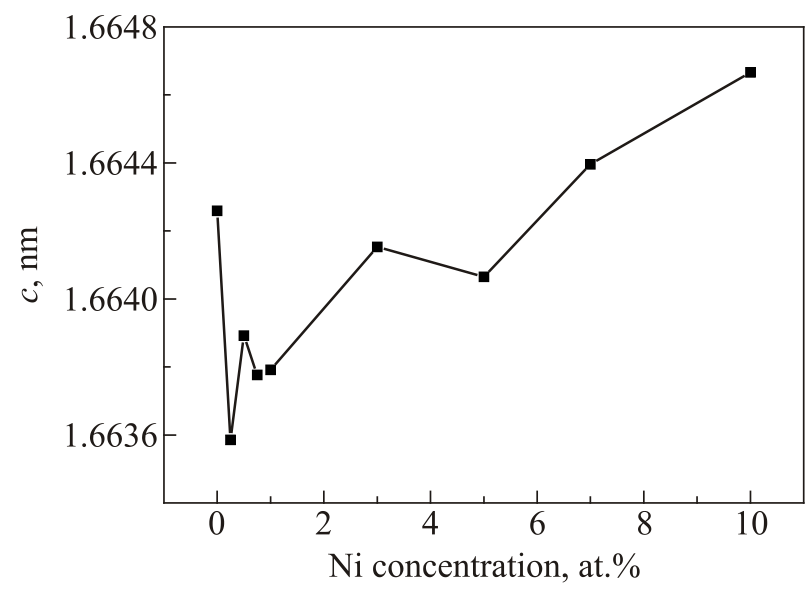

Fig. 3. Dependence of $c$ lattice parameter in $\mathrm{Ni}_{x} \mathrm{InSe}$ on nickel concentration.

The absence of diffraction maxima corresponding to metallic nickel reflects the priority-driven influence of one of the main cross-linking factors which are deformation fields. They are caused by noncoordination of the lattice constant of the crystal and the intercalant layer that assists the creation of discontinuous nanoislands formed by guest component, their reproduction with fractal nets creation etc. Thus, nickel atoms, which could insert into covalent Se-In-In-Se layers with some probability are dropped out into van der Waals gap while crystal transition to the equilibrium state. It is adjusted by a theoretical model of self purification, proposed in [28]. The concentration dependence of lattice parameter $c$ is shown in Fig. 3. Lattice parameter $a$ is independent on the concentration of the inserted nickel.

The heat capacity in the temperature range $0.3-300 \mathrm{~K}$ in magnetic field of 0 and $9 \mathrm{~T}$ has been measured using the Quantum Design PPMS-9 device.

For theoretical explanation of low-temperature heat capacity of intercalated indium selenide, a semiphenomenological approach based on the description of heat capacity in the Debye approximation was used.

\section{Experimental results}

The heat capacity of InSe and its intercalated variant $\mathrm{Ni}_{x} \mathrm{InSe}$ with concentrations $x=0.25,0.5,0.75,1,3,5,7$ and 10 at. $\%$ in the temperature range $0.3-30 \mathrm{~K}$ is shown in Fig. 4. For both pure and intercalated systems the heat capacity in this temperature region is featureless, no signs of anomalies corresponding to phase transition can be seen. For all concentrations, the heat capacity increases with temperature increase. Magnetic field up to $9 \mathrm{~T}$ has no influence on heat capacity of all intercalated compounds. For illustration, in the inset of Fig. 4, the heat capacity of $\mathrm{Ni}_{0.01}$ InSe at magnetic field of 0 and $9 \mathrm{~T}$ is shown.

The main difference between heat capacity of nonintercalated and intercalated InSe is only in shift of heat capaci-

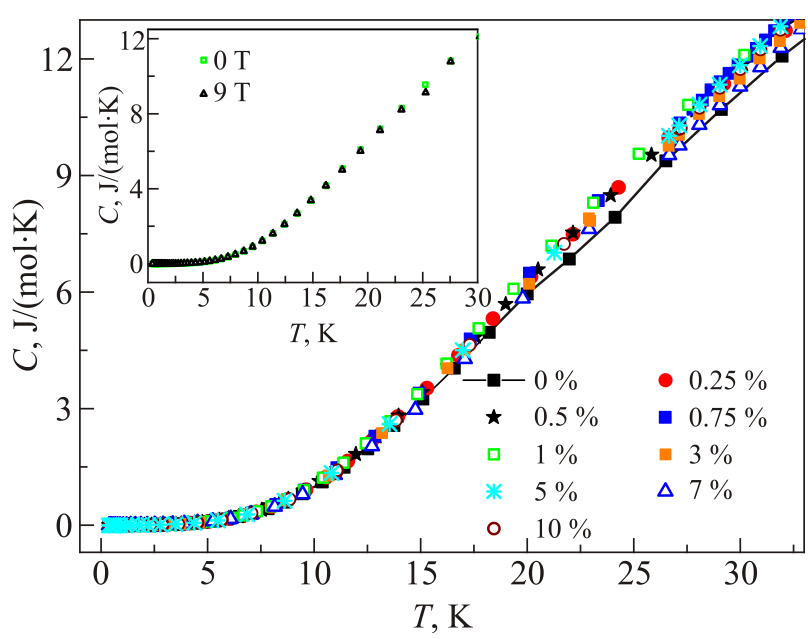

Fig. 4. (Color online) Temperature dependence of heat capacity of InSe and intercalated $\mathrm{Ni}_{x} \mathrm{InSe}$ with $x=0.25-10$ at.\% in range of $0.3-30 \mathrm{~K}$. Inset: Heat capacity of $\mathrm{Ni}_{x}$ InSe with $x=1$ at. $\%$ at magnetic field of 0 and $9 \mathrm{~T}$.

ty curves. The intercalation by nickel enhances heat capacity of $\mathrm{Ni}_{x} \mathrm{InSe}$ for all concentrations except $x=7$ at.\% at which heat capacity is reduced.

As it is known, InSe system is an insulator and nonmagnetic system at low temperatures. Thus, total heat capacity originates from the contribution of phonon subsystem and is expressed by equation $C=C_{\mathrm{ph}}$. At the lowest temperature (below $3 \mathrm{~K}$ ), heat capacity is in accordance with the Debye model and $C_{\mathrm{ph}}$ follows $T^{3}$ dependence, similarly like in Ref. 30. After intercalation of InSe by nickel atom a change in the phonon subsystem or an additional contribution to heat capacity (both electrical or magnetic) could be expected.

The total heat capacity of all intercalated $\mathrm{Ni}_{x} \mathrm{InSe}$ samples was approximated by formula containing phonon and electron term, $C=A T+\beta T^{3}$. This expression describes the experimental data of heat capacity of $\mathrm{Ni}_{x}$ InSe only in

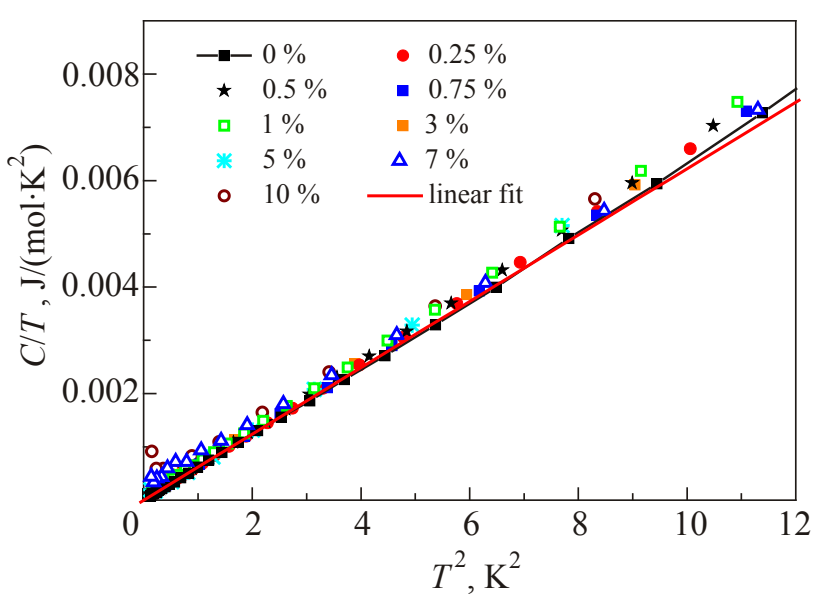

Fig. 5. (Color online) The dependence of $C / T$ on $T^{2}$ for $\mathrm{Ni}_{x} \mathrm{InSe}$ with $x=0-10$ at.\% in $0.3-3.5 \mathrm{~K}$ temperature range and a linear fit. 
relative narrow range $0.3-3 \mathrm{~K}$ (Fig. 5). In our case, linear term observed below $3 \mathrm{~K}$ is probably a combination of two contributions: the first one from the electron subsystem and the second one from the tunnelling effect proposed by Phillips and Anderson [31,32], which is usually observed in the heat capacity of disordered systems at low temperatures. Cubic term corresponds to the heat capacity of the phonon subsystem in the simple Debye approximation. To simplify the analysis we separated the contribution of linear term $A T$ from the total heat capacity, because for example this contribution to total heat capacity at $10 \mathrm{~K}$ is only $0.16 \%$ (for sample with $x=10$ at.\%).

Let us focus our attention to the issue of how the intercalation by $\mathrm{Ni}$ influences the phonon heat capacity of $\mathrm{Ni}_{x} \mathrm{InSe}$. Figure 6 shows the $\mathrm{Ni}_{x} \mathrm{InSe}$ heat capacity without linear term as $(C-A T) / T^{3}$ vs. $T$. Such representation of the heat capacity is usually used for visualization of nonDebye excess in the heat capacity related to the BP. For all compounds, a broad peak with maximum around $12.5 \mathrm{~K}$ was observed.

The maximum appearing in such a plot is in literature interpreted as BP. To our opinion, the appearance of the maximum only indicates the restrictions for using the Debye approximation. Such restrictions are caused by (in particular for such complex structures as InSe) a strong temperature dependence of the Debye temperature in lowtemperature region and also by further reasons not connected with BP. It is clear from Fig. 6, that maximum appears even for pure InSe in which the boson peak cannot appear. We may add, that for such complex structures as InSe the temperature range, in which $C$ is proportional to $T^{3}$ is very narrow.

Therefore, for the analysis of the phonon heat capacity, the $\Delta C_{\mathrm{ph}} / C_{\mathrm{ph}}$ vs. $T$ dependence was constructed for all the intercalated systems, where $\Delta C_{\mathrm{ph}}$ is the difference in phonon heat capacity between intercalated and pure InSe, and $C_{\mathrm{ph}}$ represents $C_{\mathrm{InSe}}$. Resulting dependences of $\Delta C_{\mathrm{ph}} / C_{\mathrm{ph}}$

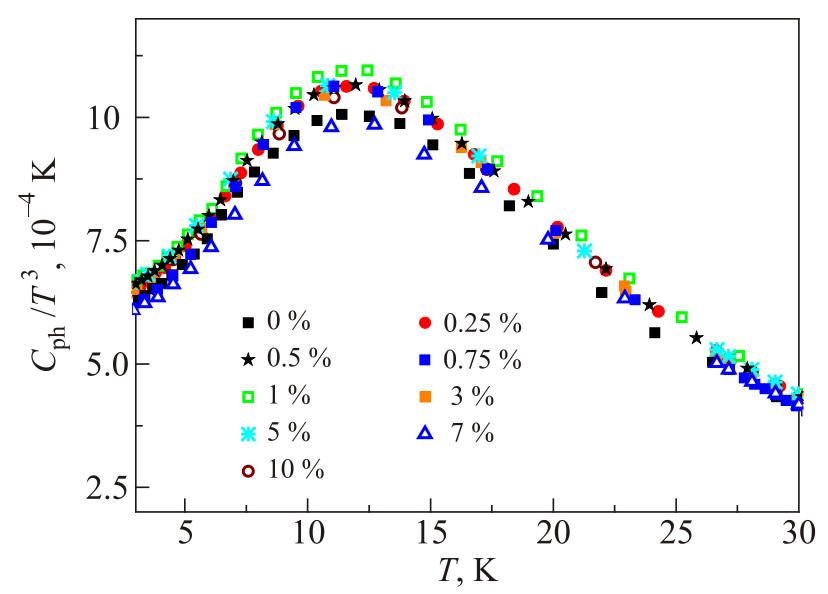

Fig. 6. (Color online) Temperature dependence of $\mathrm{Ni}_{x} \mathrm{InSe}$ ( $x=0-10$ at.\%) phonon heat capacity as $C / T^{3}$.

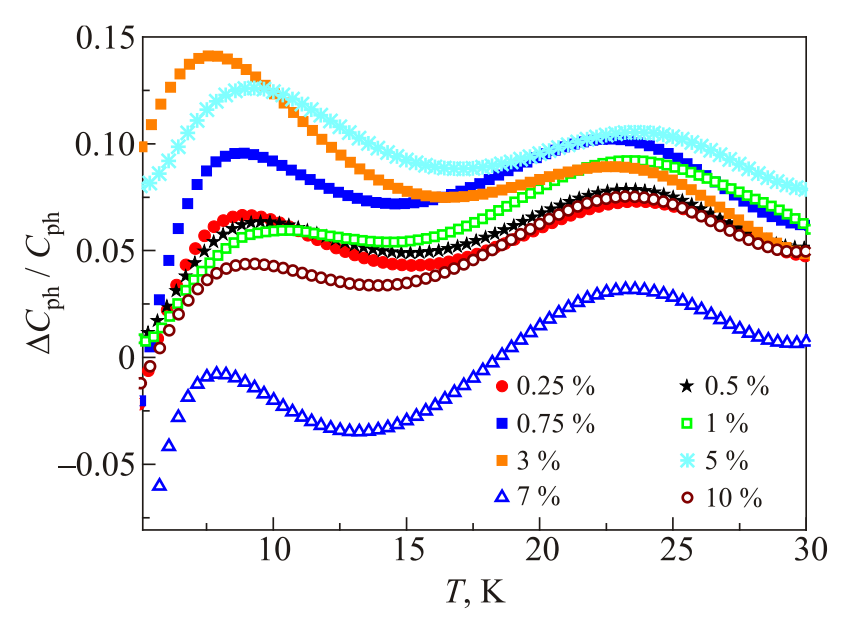

Fig. 7. (Color online) Temperature dependence of relative change of $\mathrm{Ni}_{x} \mathrm{InSe}$ phonon heat capacity $(x=0.25-10$ at.\%).

vs. $T$ for all intercalated samples are shown in Fig. 7. Two peaks in $\Delta C_{\mathrm{ph}} / C_{\mathrm{ph}}$ vs. $T$ are visible. The first one was observed at the temperature about $8 \mathrm{~K}$, and the second one was obtained at about $23 \mathrm{~K}$.

The position of both peaks are almost independent of $\mathrm{Ni}$ intercalation. An anomalous behavior was observed for a sample with concentration $x=7$ at. $\%$ which shows, unlike all other concentrations, negative values of $\Delta C_{\mathrm{ph}} / C_{\mathrm{ph}}$ vs. $T$ below approximately $20 \mathrm{~K}$.

\section{Theoretical model and calculation}

As it was illustrated in the discussion of phonon spectra of niobium diselenide [33,34], the equilibrium interatomic distance between selenium atoms (i.e., the interatomic distance corresponding to the minimum of potential which describes van der Waals interaction of selenium atoms) is equal to the distance between the closest to each other selenium atoms lying on the opposite sides of van der Waals gap, which is $\sim 3.5 \AA$. This distance between Se atoms does not correspond to the equilibrium state characterized by the minimum of the interaction potential between these atoms. The lattice stability is provided by following factors:

i) interaction between In and Se atoms which are not the nearest neighbors;

ii) interaction of atoms in "sandwiches" through the van der Waals gap separating them.

Therefore, intercalation by any impurity atoms into the van der Waals gap of InSe will inevitably lead to reconstruction. Besides, it will not only change the force constants describing the interaction between the atoms in the van der Waals gap shores (as it happens in graphite or niobium diselenide [35]), but will also of describe the interlayer interaction between atoms in "sandwich" (both the interlayer and intralayer interaction). The reconstruction of the structure and interatomic interactions may be different for different concentrations of the intercalant, which casts doubt on the possibility of restoring the force constants ac- 
cording to acoustic, optical, and neutron experiments. Therefore, it is reasonable to implement a semiphenomenological approach based on the description of heat capacity in Debye approximation, modified in [26] to better describe the exact low-temperature heat capacity for explaining the results of low-temperature heat capacity of intercalated InSe.

Debye theory is based on an approximation of the phonon spectrum of crystal acoustic waves with a linear dispersion. Indeed, at low temperatures the main contribution to phonon heat capacity is given by low frequency that is, the long-wavelength phonons, which dispersion is linear. However, numerous experimental and theoretical data show the fact that $\Theta_{D}(T)$ essentially depends on temperature and the strongest dependence of $\Theta_{D}(T)$ is observed at low temperatures.

As it is shown in [26] that the deviation of temperature dependence of heat capacity from Debye one is contribution of localized states, due to "disappearance" of acoustic phonons at the frequency of first Van Hove singularity $\omega^{*} \sim \omega_{D} / 2$. In [26] a formula for an adequate quantitative description of the low-temperature heat capacity of the crystals was also proposed

$$
\begin{gathered}
C_{v}(T)=3 R \int_{0}^{\omega_{D}}\left(\frac{\hbar \omega}{2 k T}\right)^{2} \sinh ^{-2}\left(\frac{\hbar \omega}{2 k T}\right) \times \\
\times\left\{(1-\mu) v_{D}(\omega)+\mu \delta\left(\omega-\omega^{*}\right)\right\} d \omega,
\end{gathered}
$$

where $v_{D}(\omega) \equiv 3 \omega^{2} / \omega_{D}^{3}$ is the Debye distribution function, $\mu \sim 0.2$.

For a more accurate description of low-temperature heat capacity in crystals with considerable anisotropy of elastic modulus and force constants, it is necessary to consider two "different" Debye temperatures, one for the vibrations polarized along the direction of strong bonds and the other one in the direction of weak bonds. In case of InSe, whose fibers are closely packed in a triangular lattice (1), we use $\tilde{v}_{D}(\omega)$ instead of $v_{D}(\omega)$, where $\tilde{v}_{D}(\omega)$ has the form

$$
\tilde{v}_{D}(\omega)=\frac{2}{3} v_{D}^{(\|)}(\omega)+\frac{1}{3} v_{D}^{(\perp)}(\omega)=\frac{2 \omega}{\omega_{\|}^{3}}+\frac{\omega}{\omega_{\perp}^{3}},
$$

where and $\omega_{\perp}$ values can be determined by elastic modulus of the crystal. Using data from [30] $C_{11} \approx 7.3 \cdot 10^{10} \mathrm{~Pa}, C_{12} \approx 2.7 \cdot 10^{10} \mathrm{~Pa}, C_{13} \approx 3.0 \cdot 10^{10} \mathrm{~Pa}$, $C_{33} \approx 3.6 \cdot 10^{10} \mathrm{~Pa}, C_{44} \approx 1.2 \cdot 10^{10} \mathrm{~Pa}$, as well as data on the Debye temperature of pure indium selenide $\left(\Theta_{D}^{(0)}=\sqrt[3]{3 \Theta_{\|} \Theta_{\perp} /\left(\Theta_{\|}+2 \Theta_{\perp}\right)} \approx 286 \mathrm{~K}\right)$, for InSe to obtain $\Theta_{\|}^{(0)} \approx 301.6 \mathrm{~K}, \Theta_{\perp}^{(0)} \approx 262.4 \mathrm{~K}$. The function

$$
\begin{gathered}
\tilde{v}_{0}(\omega)=\frac{2}{3} v^{(\|)}(\omega)+\frac{1}{3} v^{(\perp)}(\omega)= \\
=0.8\left(\frac{2 \omega}{\omega_{\|}^{3}}+\frac{\omega}{\omega_{\perp}^{3}}\right)+0.2\left[\frac{2}{3} \delta\left(\frac{\omega_{\|}}{2}\right)+\frac{2}{3} \delta\left(\frac{\omega_{\perp}}{2}\right)\right]
\end{gathered}
$$

is shown in Fig. 8 (green lines 1 ).

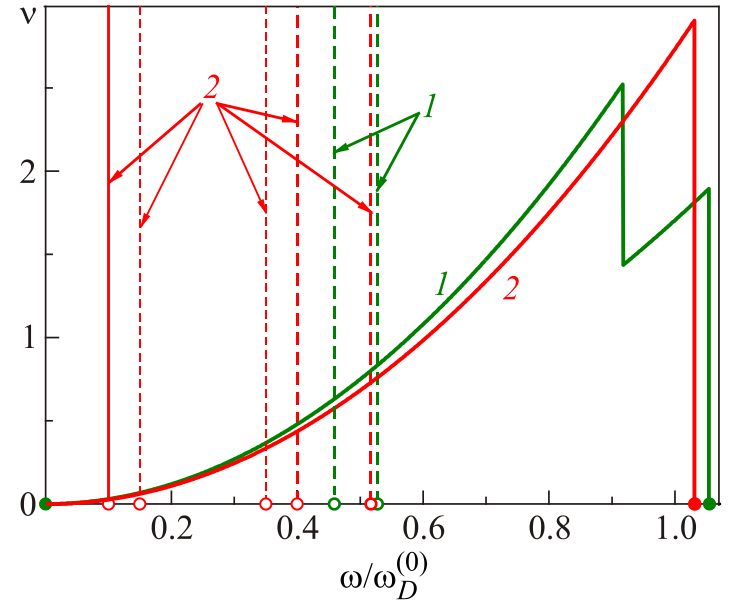

Fig. 8. (Color online) Approximation of phonon densities and combinations of Debye and Einstein distribution functions (see text).

Intercalation leads to some isotropization of indium selenide, and in addition, there is a range of sharp resonance levels corresponding to vibrations of impure atoms along different crystallographic directions (at low concentrations of the intercalant). The contribution of these levels is additive. The red lines (lines 2) in Fig. 8 show the function

$$
\begin{aligned}
& \boldsymbol{v}_{\text {inter }}(\omega)=\frac{2}{3} v_{\text {inter }}^{(\|)}(\omega)+\frac{1}{3} v_{\text {inter }}^{(\perp)}(\omega)= \\
& =0.8 \frac{3 \omega}{\tilde{\omega}_{D}^{3}}+0.2 \delta\left(\frac{\tilde{\omega}_{D}}{2}\right)+\sum_{i} \tilde{\mu}_{i} \delta\left(\frac{\tilde{\omega}_{i}}{2}\right),
\end{aligned}
$$

where $\tilde{\Theta}_{D} \equiv \hbar \tilde{\omega}_{D} / k \approx 295 \mathrm{~K}$, and the magnitudes $\tilde{\mu}_{i}$ and $\tilde{\omega}_{i}$ characterizing additive discrete levels are given in Table 1.

Table 1. Magnitudes $\tilde{\mu}_{i}$ and $\tilde{\omega}_{i}$ characterizing additive discrete levels

\begin{tabular}{c|c|c|c|c}
\hline$\tilde{\omega}_{i} / \Theta_{D}^{(0)}$ & 0.1 & 0.15 & 0.35 & 0.4 \\
\hline \hline$\tilde{\mu}_{i}, p=3$ at.\% & $6 \cdot 10^{-4}$ & $2 \cdot 10^{-3}$ & 0 & $2 \cdot 10^{-2}$ \\
$\tilde{\mu}_{i}, p=7$ at.\% & $3.5 \cdot 10^{-4}$ & $2.3 \cdot 10^{-3}$ & $2 \cdot 10^{-3}$ & $1.8 \cdot 10^{-2}$ \\
\hline \hline
\end{tabular}

Note that the closely disposed discrete energy levels in the band within the quasi-continuous spectrum indicate presence of a sharp resonance peak of finite width in the spectrum. Such maxima at very small impurity concentrations are well known as quasi-localized vibrations (see, e.g., [36]). With the increasing concentration $x$ (in our experiment $x=3$ and 7 at.\%), the scattering of long-wavelength acoustic phonons on quasi-localized vibrations levels of the impurity atoms can be interpreted as the formation of a so-called boson peak $[18,26,27,37]$ in the phonon spectrum. Note that the formation of BP in the phonon spectrum of even much simpler compounds (e.g., solid solution $\mathrm{Kr}-\mathrm{Ar}$ ) can be lead to a multiextremal dependence $\Delta C_{\mathrm{ph}} / C_{\mathrm{ph}}[25]$. 


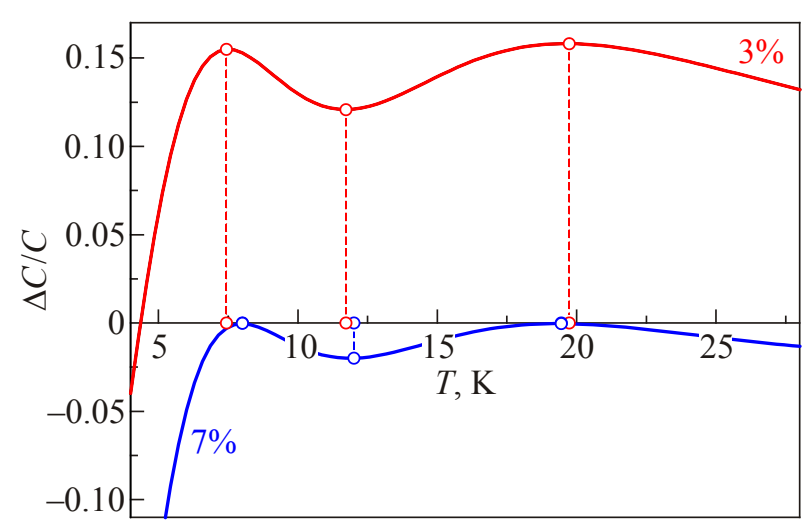

Fig. 9. (Color online) Relative change of heat capacity for intercalated InSe.

In our case the intercalation of InSe by nickel influences a large number of force constants, leading to more extremes in the temperature dependence of the relative change of the heat capacity. The broadening of these resonance peaks with increasing concentration can be described as the emergence of new discrete levels near the existing ones and as a reduction of the intensity of already existing discrete levels.

Figure 9 shows the ratio $\Delta C_{\mathrm{ph}} / C_{\mathrm{ph}}$ corresponding to phonon densities shown in Fig. 8. Thus, the presence of several extremes on the relative change in the heat capacity of InSe in case of nickel intercalation can be explained by the emergence of quasi-localized atomic vibrations of the intercalant and their scattering on the long-wavelength acoustic phonons connected mainly with atomic movement in the crystal matrix.

\section{Conclusion}

The total heat capacity of the InSe system with $\mathrm{Ni}$ impurities has been measured in magnetic field. Ni concentration was changed from 0 to 10 at. $\%$ and the magnetic field was varied from 0 to $9 \mathrm{~T}$. The absence of the phase transitions and a magnetic contribution to total heat capacity was shown. The low-temperature behavior of the phonon heat capacity of InSe intercalated by nickel has been analyzed. The experimental results show that the intercalation of nickel atoms into the van der Waals gap of InSe leads to the changes in the phonon subsystem of InSe. The changes in the phonon heat capacity caused by intercalation were theoretically explained. It has been proposed that such an intercalation of InSe by nickel atoms leads to the reconfiguration and change of the force constants describing the interaction between atoms in the van der Waals gap boundaries and also the interlayer interaction between the atoms in a "sandwich" (both for interlayer and intralayer interactions).

The observation of two peaks in the relative change of the intercalated InSe heat capacity can be explained by the emergence of quasi-local atomic vibrations of the inter- calant and by their scattering on the long-wavelength acoustic phonons connected mainly with the movement of atoms in the crystal matrix. It is these facts which lead to the formation of a boson peak manifested in multiextremal temperature dependence of $\Delta C_{\mathrm{ph}} / C_{\mathrm{ph}}$ in studied systems.

\section{Acknowledgments}

We thank M.I. Bagatskii for critical reading of the manuscript and useful discussions.

This work is the result of the implementation of the project Research and Education at UPJŠ - Heading towards Excellent European Universities, ITMS project code 26110230056, supported by the Operational Program Education funded by the European Social Fund (ESF). This work was also supported by the project of Grant Agency VEGA, grant No. 1/0145/13 and by the grant of the Ukrainian Academy of Sciences under the contract No. 4/13-N "Quantum phenomena in the nanosystems and nanomaterials at low temperatures".

1. I.V. Stasyuk and Yu.I. Dublenych, Phys. Rev. B 72, 224209 (2005).

2. Y.M. Stakhira, N.K. Tovstyuk, V.L. Fomenko, I.I. Grigorchak, A.K. Borysyuk, and B.A. Seredyuk, Fiz. Nizk. Temp. 38, 69 (2012) [Low Temp. Phys. 38, 54 (2012)].

3. Modern Battery Technology, C.D.S. Tuck (ed.), Ellis Horwook, New York (1991).

4. S.A. Voitovych, I.I. Grygorchak, and O.I. Aksimentyeva, Mol. Cryst. Liq. Cryst. 497, 55 (2008).

5. F.R. Gamble, F.J. DiSalvo, R.A. Klemm, and T.H. Geballe, Science 168, 568 (1970).

6. K. Zaghib and R. Kinoshita, in: NATO Science Series "New Trends in Intercalation Compounds for Energy Storage", C. Julien, J.P. Pereira-Ramos, and A. Momchilov (eds.), Springer (2002).

7. Z. Sobiesierski and J.B. Clegg, Appl. Phys. Lett. 63, 926 (1993).

8. I.M. Stakhira, N.K. Tovstyuk, V.L. Fomenko, V.M. Tsmots, and A.N. Shchupliak, Semiconductors 45, 1258 (2011).

9. U. Buchenau, A. Wischnewski, M. Ohl, and E. Fabiani, J. Phys.: Condens. Matter 19, 205106 (2007).

10. H. Cang, J. Li, H.C. Andersen, and M.D. Fayer, J. Chem. Phys. 123, 064508 (2005).

11. M. Foret, E. Courtens, R. Vacher, and J.-B. Suck, Phys. Rev. Lett. 77, 3831 (1996).

12. G. Baldi, V.M. Giordano, G. Monaco, and B. Ruta, Phys. Rev. Lett. 104, 195501 (2010).

13. M. Naftaly and R.E. Miles, J. Non-Cryst. Solids 351, 3341 (2005).

14. G. Baldi, A. Fontana, G. Monaco, L. Orsingher, S. Rols, F. Rossi, and B. Ruta, Phys. Rev. Lett. 102, 195502 (2009).

15. M.B. Tang, H.Y. Bai, and W.H. Bang, Phys. Rev. B 72, 012202 (2005).

16. Y. Li, H.Y. Bai, W.H. Wang, and K. Samwer, Phys. Rev. B 74, 052201 (2005). 
17. M. Hassaine, M.A. Ramos, A.I. Krivchikov, I.V. Sharapova, O.A. Korolyuk, and R.J. Jiménez-Riobóo, Phys. Rev. B 85, 104206 (2012).

18. B.B. Laird and H.R. Schober, Phys. Rev. Lett. 66, 636 (1991).

19. S.R. Elliott, Europhys. Lett. 19, 201 (1992).

20. W. Schirmacher, G. Diezemann, and C. Ganter, Phys. Rev. Lett. 81, 136 (1998).

21. W. Schirmacher, Phys. Status Solidi B 250, 937 (2013).

22. V.L. Gurevich, D.A. Parshin, and H.R. Schober, JETP Lett. 76, 553 (2002).

23. V.L. Gurevich, D.A. Parshin, and H.R. Schober, Phys. Rev. $B$ 67, 094203 (2003).

24. V. Gurarie and A. Altland, Phys. Rev. Lett. 94, 245502 (2005).

25. M.I. Bagatskii, S.B. Feodosyev, I.A. Gospodarev, O.V. Kotlyar, E.V. Manzhelii, A.V. Nedzvetski, and E.S. Syrkin, Fiz. Nizk. Temp. 33, 741 (2007) [Low Temp. Phys. 33, 564 (2007)].

26. I.A. Gospodarev, V.I. Grishaev, A.V. Kotlyar, K.V. Kravchenko, E.V. Manzheli, E.S. Syrkin, and S.B. Feodosyev, Fiz. Nizk. Temp. 34, 829 (2008) [Low Temp. Phys. 34, 655 (2008)].

27. T. Bryk and I. Mryglod, Phys. Rev. B 82, 174205 (2010).

28. K.D. Tovstyuk, Semiconductor Materials Science, Naukova Dumka, Kiev (1984) (In Russian).

29. A.I. Dmitriev, G.V. Lashkarev, P.E. Butorin, V.I. Sichkovskyi, M.V. Radchenko, Z.D. Kovalyuk, A.I. Beskrovni,
P. Aleshkevych, R. Szymczak, D. Dobrovolski, and R. Minikaev, Ukr. J. Phys. 53, 562 (2008).

30. K. Imai, K. Kumazaki, S. Seto, K. Yamaya, and Y. Abe, J. Chem. Sci. 102, 601 (1990).

31. P.W. Anderson, B.I. Halperin, and C.M. Varma, Philos. Mag. 25, 1 (1972); W.A. Phillips, J. Low Temp. Phys. 7, 351 (1972).

32. Amorphous Solids: Low Temperature Properties, W.A. Phillips (ed.), Springer, Berlin (1981); Rep. Prog. Phys. 50, 1657 (1987).

33. I.K. Galetich, I.A. Gospodarev, V.I. Grishaev, A.V. Eremenko, K.V. Kravchenko, V.A. Sirenko, and S.B. Feodosyev, Superlattices Microstruct. 45, 564 (2009).

34. I.A. Gospodarev, V.V. Eremenko, K.V. Kravchenko, V.A. Sirenko, E.S. Syrkin, and S.B. Feodosyev, Fiz. Nizk. Temp. 36, 436 (2010) [Low Temp. Phys. 36, 344 (2010)].

35. A. Feher, I.A. Gospodarev, V.I. Grishaev, K.V. Kravchenko, E.V. Manzheli, E.S. Syrkin, and S.B. Feodosyev, Fiz. Nizk. Temp. 35, 862 (2009) [Low Temp. Phys. 35, 679 (2009)].

36. A.M. Kosevich, The Crystal Lattice, Phonons, Solitons, Dislocations, Superlattices, WILEY-VCH, Verlag, Berlin, GmBH, Berlin (1999), p. 345.

37. A. Feher, E.S. Syrkin, S.B. Feodosyev, I.A. Gospodarev, E.V. Manzhelii, A.V. Kotlyar, and K.V. Kravchenko, in: Waves in Fluids and Solids, R.P. Vila (ed.), InTech, Rijeka (2011). 